\title{
Interfaces
}

INTERFACES Image Texte Language

$45 \mid 2021$

Jeux de formats (1)

\section{Jan Baetens, Une fille comme toi, Ciné-Roman-Photo}

\author{
Isabelle Schmitt-Pitiot
}

\section{(2) OpenEdition}

Journals

Édition électronique

URL : https://journals.openedition.org/interfaces/2985

DOI : 10.4000/interfaces.2985

ISSN : 2647-6754

Éditeur :

Université de Bourgogne, Université de Paris, College of the Holy Cross

Édition imprimée

Date de publication : 12 juillet 2021

ISSN : 1164-6225

\section{Référence électronique}

Isabelle Schmitt-Pitiot, « Jan Baetens, Une fille comme toi, Ciné-Roman-Photo », Interfaces [En ligne], 45 | 2021, mis en ligne le 12 juillet 2021, consulté le 16 septembre 2021. URL : http://

journals.openedition.org/interfaces/2985; DOI : https://doi.org/10.4000/interfaces.2985

Ce document a été généré automatiquement le 16 septembre 2021.

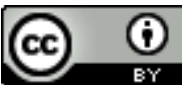

Les contenus de la revue Interfaces sont mis à disposition selon les termes de la Licence Creative Commons Attribution 4.0 International. 


\title{
Jan Baetens, Une fille comme toi, Ciné- Roman-Photo
}

\author{
Isabelle Schmitt-Pitiot
}

\section{RÉFÉRENCE}

Jan Baetens, Une fille comme toi, Ciné-Roman-Photo. Paris : Jean Boîte Éditions : Paris, 2020. 48 p. ISBN : 978-2-36568-032-5.

1 Jan Baetens, poète et chercheur à l'Université de Leuven (Louvain, Belgique), historien et théoricien du ciné-roman-photo, n'est pas un inconnu aux manifestations scientifiques à l'initiative de la revue Interfaces. En effet, il participa à Dijon à un colloque sur la bande dessinée et le dessin de presse en 2019, après avoir ouvert en 2012 et 2013 deux journées d'études sur "Bandes dessinées et adaptation », en tant que spécialiste de la création intermédiale. Ici, auteur et/ou réalisateur, il inscrit son travail dans un entre-deux, ou plutôt un entre-trois, comme se croisent les écritures, les lectures et les époques. 
Figure 1. Jan Baetens, Une fille comme toi, Ciné-Roman-Photo.

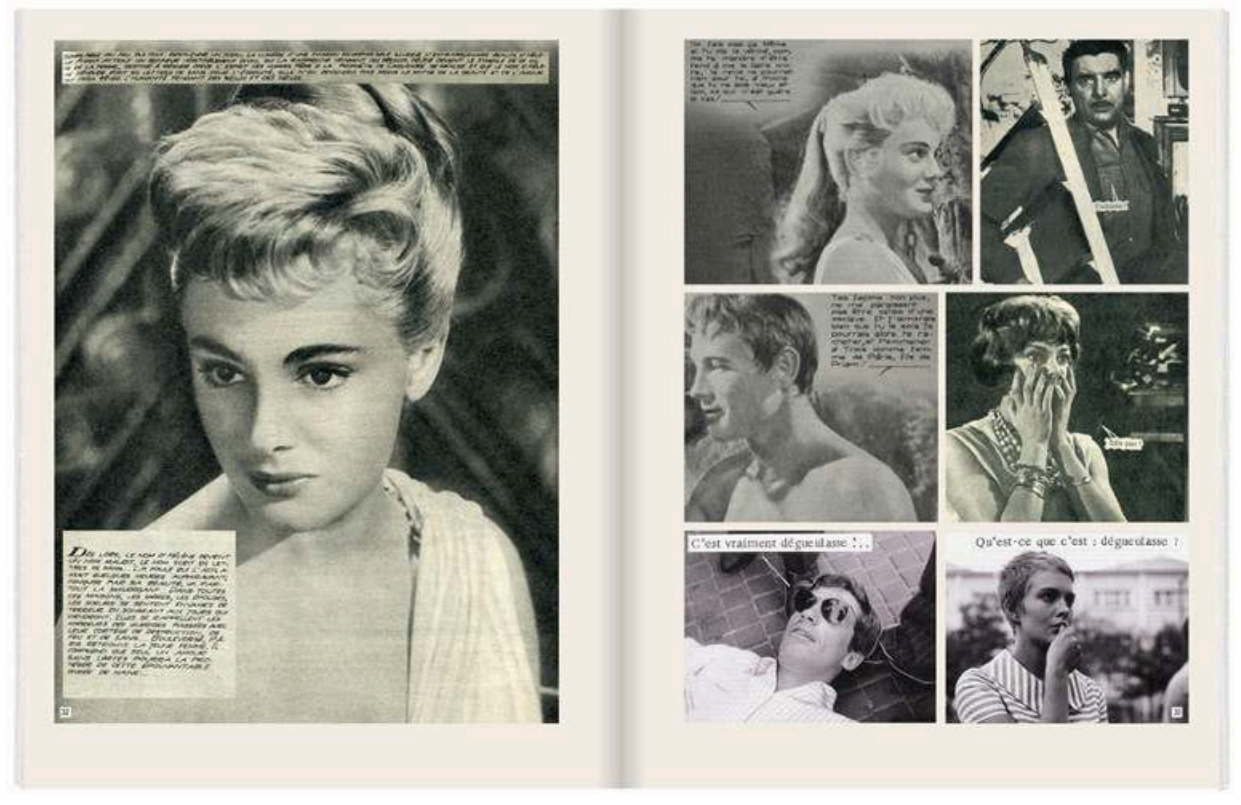

(c) Paris, JBE Books.

2 De belle qualité matérielle, pour laquelle il convient de saluer l'éditeur Jean Boîte, ce livre dit d'écriture sans écriture (ou uncreative writing), qui revendique et sert à merveille son support papier, offre à son lecteur, ou à sa lectrice, le genre importe, on le verra, une véritable aventure esthétique et intellectuelle. Le moteur en est un réseau quasi infini de contradictions et de correspondances fécondes au fil des pages tournées, à commencer par le dépouillement, tout en lettres noires sur fond blanc, de la couverture, qui s'ouvre, après l'éclair rouge de sa deuxième, sur un flamboyant portrait de Brigitte Bardot.

3 Cette page révèle la nature "en tiroir" de l'ouvrage et en condense l'essence puisqu'elle se présente comme la "vraie » couverture du ciné-roman-photo Une fille comme toi, soit la reprise d'un film sous forme de roman-photo, cinéma de papier qui fit florès entre 1955 et 1965. À la une, le regard caméra de Bardot pèse son poids de malice et de provocation, au seuil d'une création mêlant l'artifice et l'authentique, et invitant au plaisir comme au questionnement.

4 Jouant sur deux tableaux temporels, l'ouvrage alterne dans un savant feuilletage, textes de présentation, essais contemporains et reproductions de ciné-romans-photos "d'époque ». CEuvre au second degré, Une fille comme toi recrée un film qui n'a jamais existé puisque sont ici remontés 300 photogrammes tirés de plus de 1500 publications différentes. Ingénieux, le montage intrigue et séduit, en particulier si l'on possède la culture cinématographique nécessaire à la reconnaissance de dizaines de films, des plus ambitieux aux plus modestes, et d'une myriade de vedettes de l'écran, pour parler comme dans les années soixante.

5 Il nous semble cependant qu'au-delà du plaisir de la remémoration, le livre ouvre bien des pistes de réflexion. Son titre, par exemple, laisse songeur, et surtout songeuse : qui s'adresse si familièrement à toi, lectrice, t'interpelle une première fois, disons en 2020, puis remonte le temps et réitère son invite, faisant miroiter les délices de 
l'identification, avec ce qu'il se doit d'ironie dans le décalage? Dès lors, on pourra s'interroger, par exemple, sur le sexisme des romans photos comme du "ciné ", où des instances de production bien masculines cherchent à séduire un public féminin réduit à des rôles stéréotypés, et puis dépasser ce premier niveau d'analyse pour prendre en compte aussi la dimension libératrice de ces productions populaires jadis dédaignées, puis oubliées, et qui reviennent ainsi recrées et mises en perspective.

Enfin le livre se referme sur quelques critiques, dont deux, peut-être trop sévères pour ne pas être ironiques, voire, qui sait, apocryphes, l'accusent de malhonnêteté et de plagiat et posent la question de fond de la création et de l'originalité. Nous suivrons alors le conseil que le livre lui-même nous adresse en guise de malicieux au revoir: «Jugez vous-même!»

\section{AUTEURS}

\section{ISABELLE SCHMITT-PITIOT}

Université de Bourgogne-Franche-Comté - Isabelle Schmitt-Pitiot est Maître de Conférences en Études Anglophones, retraitée de l'UFR de Langues et de Communication de l'Université de Bourgogne-Franche-Comté (UB-FC) et membre honoraire du Centre de Recherche Texte-ImageLangage (TIL) de l'UB-FC. Membre de la Société d'Etudes et de Recherches sur le Cinéma Anglophone (SERCIA), elle travaille sur le cinéma et les séries télévisées anglophones, surtout nord-américaines. Elle a communiqué et publié sur les genres et la réception filmiques, sur des réalisateurs (Woody Allen, John Ford, John Huston, Robert Altman, Milos Forman) et des séries (Desperate Housewives, Sopranos, ER/Urgences, MASH). Elle a édité Intimacy in Cinema (McFarland, 2014) et De l'intime dans le cinéma anglophone (CinémAction, Corlet, 2015) avec David Roche, Bande dessinée et adaptation avec Benoît Mitaine et David Roche (Presses Universitaires Blaise Pascal, 2015) et Sur la route : quand le cinéma franchit les frontières avec Bénédicte Brémard et Julie Michot (Editions Universitaires de Dijon, 2018). Elle édite actuellement avec Julia Echeverria les numéros 7 et 8 de la revue électronique Film Journal, à paraître sous le titre Borderlines aux deuxième et troisième trimestres 2021. 